\title{
ANALYSIS OF THE INHIBITION POTENTIAL OF STREPTOCOCCUS SALIVARIUS ISOLATED FROM THE SALIVA AND DORSUM OF THE TONGUE OF ADULTS ON THE GROWTH OF STREPTOCOCCUS MUTANS
}

\section{DEBORAH CHINTYANITA, HEDIJANTI JOENOES, BOY M BACHTIAR*}

\author{
Department of Oral Biology, Faculty of Dentistry, Universitas Indonesia, Jakarta, Indonesia. Email: boybachtiar@gmail.com
} Received: 20 April 2017, Revised and Accepted: 13 July 2017

\section{ABSTRACT}

Objective: The aim of this study is to analyze the inhibitory potential of Streptococcus salivarius, after it has been isolated from saliva and the tongue's dorsum, on the growth of Streptococcus mutans.

Method: Polymerase chain reaction, SDS PAGE, Bradford test, deferred antagonism test, and well-diffusion test were used.

Result: S. salivarius isolated from saliva and the tongue's dorsum inhibited the growth of $S$. mutans, but it was not the case with S. salivarius ATCC 13419 ( $\mathrm{p}<0.05)$. However, there was no significant difference between the inhibition of $S$. mutans from $S$. salivarius isolated from saliva and the tongue's dorsum $(\mathrm{p}>0.05)$.

Conclusion: S. salivarius isolated from saliva and the tongue's dorsum can inhibit the growth of S. mutans, but there was no significant difference in the inhibitory potential of $S$. salivarius isolated from saliva on the tongue's dorsum.

Keywords: Probiotic, Streptococcus salivarius, Streptococcus mutans.

(C) 2017 The Authors. Published by Innovare Academic Sciences Pvt Ltd. This is an open accessarticle under the CC BY license (http://creativecommons. org/licenses/by/4. 0/) DOI: http://dx.doi.org/10.22159/ajpcr.2017.v10s5.23082

\section{INTRODUCTION}

Antibiotic resistance is a problem in antibiotic therapies [1]. Probiotic use is an alternative to resolve this problem. A probiotic is a living microorganism that when given in an adequate amount, will benefit its host [2]. Probiotics are commonly used in the prevention or treatment of gastrointestinal diseases and infections. However, the reports over the past few decades have showed the use of probiotics could improve oral health as well. Out of the 700 species detected in the oral cavity, Lactobacillus and Bifidobacterium are two genera that are commonly used as probiotics [3,4]. Examples of probiotics that are advantageous to oral health are Lactobacillus rhamnosus strain GG and Saccharomyces boulardii [5]. Probiotics work by inhibiting the adhesion of a pathogen to host tissues, stimulating and modulating the immune system of mucosa, modulating apoptosis during cell proliferation, repairing barrier integrity, and destroying or inhibiting the growth of pathogenic bacteria by bacteriocin production or other pathogenic bacteria antagonistic products.

Bacteriocins are antimicrobial proteins or peptides produced by bacteria. Bacteriocins can inhibit growth or kill other bacteria. A bacteriocin-like inhibitory substance (BLIS) is a natural alternative from antibiotics because it can reduce the growth of multidrug-resistant bacteria. BLIS is a protein that acts like antibiotics and has a narrow-spectrum killing activity. BLIS has been used and produced by bacteria with a bacteriocinlike inhibitory effect. Streptococcus salivarius is one of the bacteria that produces BLIS and has been studied before [6]. S. salivarius has potential BLIS and can produce a probiotic with the target of pathogenic bacteria in the oral cavity [7]. S. salivarius is known as an early colony form found on oral cavities of neonates, originated from the mother [7]. S. salivarius on a swab sample taken from neonates showed $10 \%$ of total isolated Streptococcus. This amount will keep increasing for $25-30 \%$ after 1 month. In a healthy adult, the amount of $S$. salivarius is approximately $2 \%$ from the total isolated Streptococcus from buccal mucosa, $17 \%$ from the tongue, and $30 \%$ from the pharynx. In a saliva sample, the amount of S. salivarius is approximately $10^{6}-10^{7}$ colonial units (CFU/ml) [8].
S. salivarius K12 isolated from healthy children's saliva can be used as a probiotic. This strain has antimicrobial activity against $S$. pyogenes and other bacteria that contribute to halitosis because it can produce antibiotic bacteriocin [7]. Anther salivarius strain, S. salivarius TOVE-R, was reported to be antagonistic to Streptococci, which contributed to the caries process, such as Streptococcus mutans and S. sobrinus, pharyngitis, such as $S$. pyogenes, or other pathogens involved in periodontitis [9]. Other studies investigating S. salivarius M18 showed that the bacteriocin produced was antagonistic toward S. mutans, one of the species responsible for the pathogenesis of dental caries [10]. Dental caries are the most commonly found dental problem in Indonesia [11]. The probiotic effect of S. salivarius is already known, but the inhibitory potential of the protein produced by Indonesian isolates of $S$. salivarius on the growth of $S$. mutans has not yet been discovered. This study aimed at analyzing the inhibitory potential of a protein produced by S. salivarius, which was isolated from saliva and the tongue's dorsum from healthy adult subjects, on the growth of $S$. mutans. It is possible that proteins produced by $S$. salivarius can be used as a probiotic agent to prevent dental caries in Indonesia.

\section{METHODS}

Subjects chosen were 19-21 years of age, physically healthy, and caries free. Ten subjects meeting these criteria were informed and gave their written consent to be subjects of this research. Subjects were asked not to consume high sugar, acid, and caffeine $3 \mathrm{hrs}$ before sample taking. Subjects were then instructed to gargle water for approximately 1 minute and stayed idle for 10 seconds to avoid dilution of the saliva sample.

Stimulated saliva samples were taken after subjects were instructed to chew on Parafilm M; then, saliva samples were contained in sterile vials that were then capped properly, labeled with name, date, age, and sex, and stored temporarily in a chiller box. Swab samples from the dorsum of the tongue were taken after tongue isolation with a sterile cotton roll. Swab samples were taken using cytobrush in the circumvallate papillae 
area until the tip of the tongue. Then, the cytobrush containing saliva was contained in a vial with PBS (pH 7.2), labeled with name, date, age, and sex, and stored temporarily in a chiller box.

Saliva and tongue swab samples and S. salivarius ATCC 13419 were cultured on a Mitis Salivarius Agar medium (MSA) for $24 \mathrm{hrs}$ inside a $37^{\circ} \mathrm{C}$ incubator. Identification of colony morphology was conducted by observing the size and consistency of the surface (big, soft, smooth, and mucous-like colony that looks like a gum drop) [12]. Colonies considered as S. salivarius were taken and confirmed using the polymerase chain reaction (PCR) technique with SalAUS primers (5'-GTAGAAAATATTTACTACATACT) and SalADS (5'-GTTAAAGTATTCGTAAAACTGATG) [13]. PCR amplification results were analyzed using $1 \%$ agarose running on electrophoresis for 30 minutes (100V) [13]. DNA strands that were $118 \mathrm{bp}$ indicated that the colonies identified on the MSA medium were $S$. salivarius. Those colonies were taken as stocks in $30 \%$ glycerol, stored in a freezer with a temperature of $-80^{\circ} \mathrm{C}$, and given back on $\mathrm{BHI}$ agar and liquid medium (stored in anaerobic jar containing mixed gases and put into an incubator for $18 \mathrm{hrs}$ ).

In a liquid culture medium, the cell lysate method was used to obtain whole-cell protein, and centrifugation was used to obtain proteins in the spent medium. Whole-cell and spent-medium proteins were analyzed using SDS PAGE (150 V, $80 \mathrm{~mA}, 60$ minutes) and given the color "Coomassie Blue." The SDS-PAGE test showed four subjects with similar protein profiles, $70 \mathrm{kDa}, 40 \mathrm{kDa}$, and $10 \mathrm{kDa}$ strands, as shown in Fig. 1. Proteins with similar molecule masses were measured for the concentration with Bradford method [14]. Then, S. salivarius colonies with these similar molecule masses were taken and stored on an Eppendorf tube containing 30\% glycerol and stored in a freezer with a temperature of $-80^{\circ} \mathrm{C}$.

Clinical and ATCC 13419 S. salivarius were diluted onto four different concentrations $(100 \%, 10 \%, 1 \%$, and $0.1 \%)$. Each concentration was scratched on a $1 \mathrm{~cm}$ wide rectangle area on an agar BHI plate. The agar plate was then put inside an anaerobic jar, given a mix of gases, and incubated at $37^{\circ} \mathrm{C}$ for $24 \mathrm{hrs}$. Subsequently, bacteria seen macroscopically on the agar surface were removed by swabbing with a glass object. The agar surface was exposed to chloroform for 30 minutes by putting the plate upside down toward filter paper, which was immersed in chloroform, to kill bacteria on the surface. After 30 minutes, the plate was left for 15 minutes to remove chloroform residue. S. mutans XC were cultured in an agar BHI medium for $24 \mathrm{hrs}$. One colony of cultured S. mutans was taken and put inside an Eppendorf tube containing soft agar, which was then vortexed until the bacteria were mixed with the soft agar. S. mutans mixed with the soft agar were taken using a cotton swab and swabbed perpendicular with the S. salivarius swab, which had been cleaned. This procedure was done Duplo. Then, the plate was put inside an anaerobic jar, given mixed gases, and incubated at $37^{\circ} \mathrm{C}$ for $24 \mathrm{hrs}$. Subsequently, the inhibitory zone of $S$. mutans growth was checked.

Two loopfuls of $S$. mutans XC colonies were taken and put inside an Eppendorf tube containing PBS and then vortexed until homogenous. $100 \mathrm{ml}$ of the agar BHI were made and sterilized. Then, S. mutans that have been diluted in PBS before were mixed into liquid agar BHI.

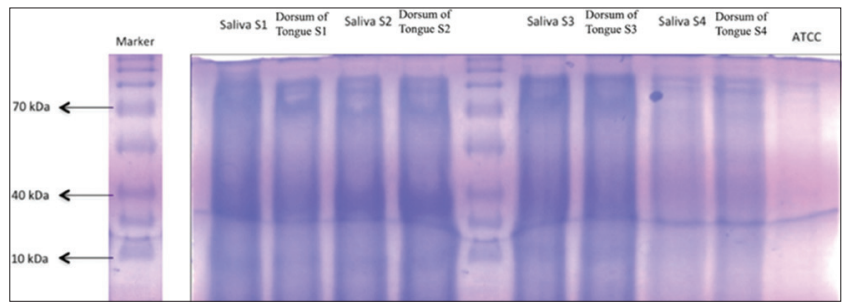

Fig. 1: Streptococcus salivarius protein profile based on SDS-PAGE analysis
Subsequently, the agar BHI was put into an orbital shaker for 30 seconds and then poured into a Petri dish. After the agar hardened, four wells were made on the agar inside each petri dish. Afterward, each protein concentration was put into a well and incubated at $37^{\circ} \mathrm{C}$ for $24 \mathrm{hrs}$. Then, the well was checked for any gap between it and the growing bacteria colony, and every gap visible was measured.

Data obtained from S. salivarius identification from healthy subjects (saliva and dorsum of tongue samples) were analyzed using the Crosstabs Chi-square test. Then, data on of the diameter showing the inhibition zone of S. salivarius and S. salivarius protein isolated from saliva and the tongue's dorsum on $S$. mutans growth were analyzed using a one-way ANOVA and t-test. Results are significantly different if $\mathrm{p} \leq 0.05$.

\section{RESULTS}

S. salivarius identification was carried out by comparing the morphology of clinical and ATCC 13419 S. salivarius that had been grown on the MSA medium. Out of the 10 subjects, 6 had similar S. salivarius colony morphologies, big, sticky, and mucous-like. The colonies of these six subjects were tested with PCR to confirm $S$. salivarius by finding fragmented strands of $118 \mathrm{bp}$. PCR results showed that colonies from all six samples were $S$. salivarius (Fig. 2).

The SDS PAGE test was conducted on the six subjects to identify the protein produced by clinical and ATCC 13419 S. salivarius. The results showed that clinical $S$. salivarius on four subjects produced proteins with identical molecular masses, $70 \mathrm{kDa}, 40 \mathrm{kDa}$, and $10 \mathrm{kDa}$. Clinical S. salivarius from those four subjects was subjected to a deferred antagonism test. Only one subject could be interpreted. Therefore, the next steps only used one subject. Measurement results from the inhibition zone are shown in Fig. 3.

A one-way ANOVA and independent sample t-test results found that the inhibition zone from ATCC 13419 was statistically significant with the inhibition zone isolated from saliva and the tongue. However, the inhibition zone from saliva samples was not significant compared to tongue samples. The protein inhibitory potential secreted by S. salivarius on S. mutans was tested using the well-diffused agar method. In Figs. 4 and 5, Tables 1 and 2, the inhibition zone means around the wells on every isolation source and on all concentration levels are shown in Figs. 3-5.

The inhibition zone was formed in the well-diffusion test on a wholecell protein of $S$. salivarius that had been isolated from saliva and the tongue's dorsum on the highest concentration, with a means of $8.25 \mathrm{~mm}$ for saliva samples and $5.75 \mathrm{~mm}$ for tongue's dorsum samples. However, the inhibition zone was not formed in lower concentrations. Meanwhile, no inhibition zone diameter was formed in all concentration of S. salivarius ATCC 13419. The inhibition zone's

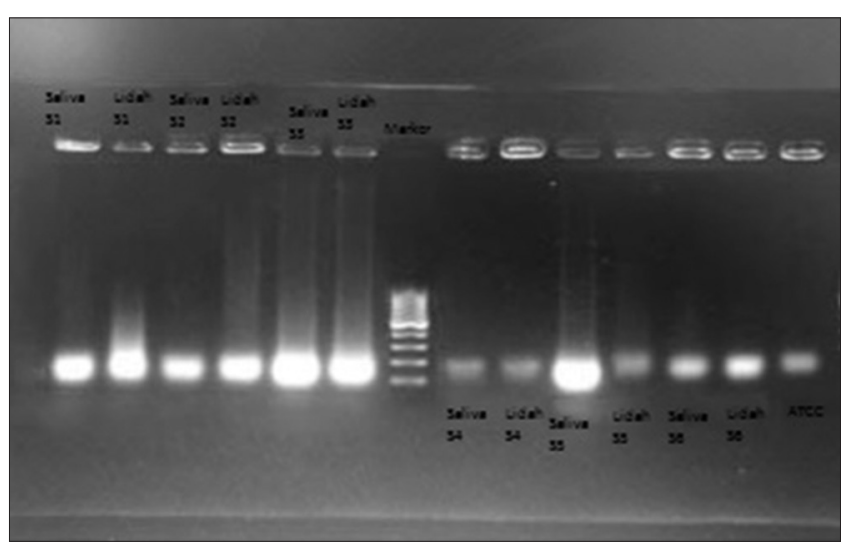

Fig. 2: Confirmation of clinically isolated Streptococcus salivarius using the polymerase chain reaction technique 
diameter was not formed and produced similar results to the control group, the $S$. salivarius ATCC 13419 spent-medium protein. The welldiffusion test on a spent-medium protein of clinical $S$. salivarius isolated from saliva and the tongue's dorsum also showed no results in the inhibition zone's diameter, but $S$. mutans XC colonies that formed around the well were smaller compared to the control or S. salivarius ATCC 13419.

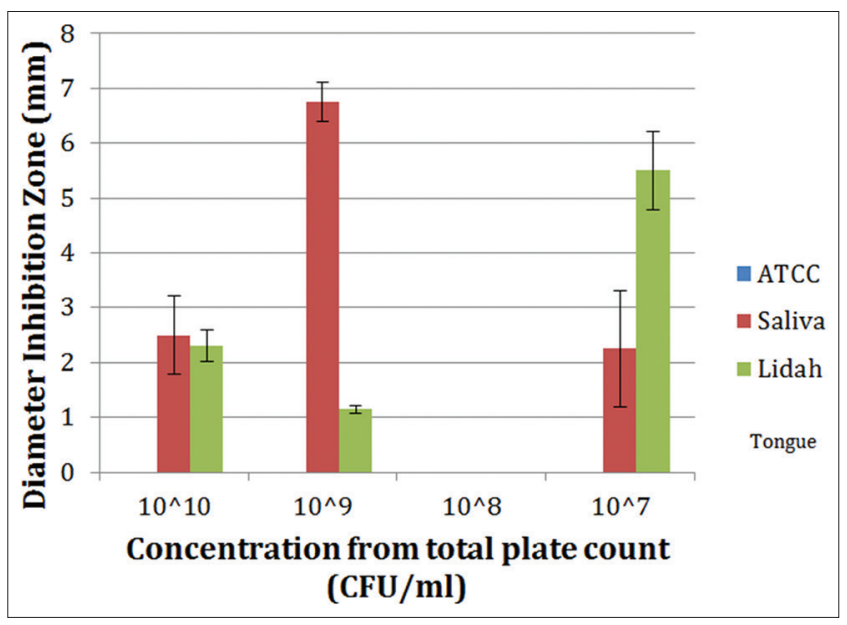

Fig. 3: Measurement means results of the inhibition zone's diameter of Streptococcus mutans XC by ATCC 13419 Streptococcus salivarius and clinical $S$. salivarius isolated from saliva and the tongue's dorsum

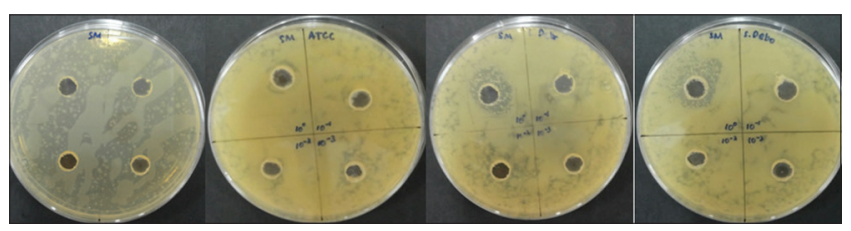

Fig. 4: Test results and control of well-diffusion test on Streptococcus salivarius ATCC 13419, dorsum of tongue isolation, and saliva isolation whole-cell protein

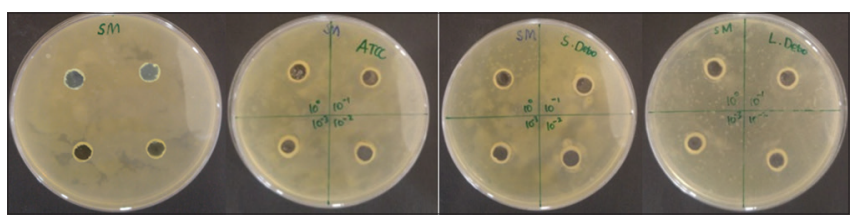

Fig. 5: Control and results from well-diffusion test on spentmedium protein of Streptococcus salivarius ATCC 13419,

$S$. salivarius isolated from saliva, and $S$. salivarius isolated from dorsum of tongue

\section{DISCUSSION}

In this study, the identification of S. salivarius isolated from the test subjects was carried out using the inclusion criteria. Out of the 10 subjects, 6 were identified as having S. salivarius in the saliva and dorsum of tongue samples. Statistical analysis showed no significant difference between both samples. This is in accordance with the theory that S. salivarius will colonize mainly in saliva and the tongue's dorsum [15]. This result is also in agreement with the research conducted by Horz that showed that $S$. salivarius was found in healthy adult subjects, both on the tongue and in saliva [8]. Nevertheless, these results contradict a study by Wescombe that indicated that $S$. salivarius is a pioneer colony in the oral cavity of humans and can survive throughout the life of the person [16]. If so, S. salivarius should be found all over the oral cavity. The reason why it may not be found could be because of the variation of the subjects' dietary habits. Food particles and debris may provide nutrition for bacteria, promoting bacterial growth. Furthermore, a study by Roger showed that saliva contained many enzymes, such as lysozyme, lactoperoxidase, and amylase, that might have antimicrobial properties [17]. These conditions may affect the growth rate of $S$. salivarius in oral cavities. Therefore, these differentiate this study from the previous studies mentioned above.

A deferred antagonism test method had been used to analyze the inhibition potential of S. salivarius against S. mutans XC growth. Research data showed that in all S. salivarius ATCC 13419 concentrations, no S. mutans XC inhibition zones formed, whereas inhibition zones formed in clinical $S$. salivarius (of concentration $10^{10}, 10^{9}$, and $10^{7} \mathrm{CFU} / \mathrm{ml}$ ). Independent sample t-tests showed that the inhibition zone formed by the saliva isolate was not significantly different than the dorsum of tongue isolate. This indicated that the inhibitory potential of clinical $S$. salivarius does not depend on the concentration. Inhibition zone formation from $S$. salivarius isolated from saliva and the tongue's dorsum was in agreement with the study conducted by Di Pierro, where S. salivarius M18 made into oral tablets as a nutrition supplement (Carioblis) was used on children 6-17 years old with high caries risk based on cariograms taken before the research began [10]. In $\mathrm{Di}$ Pierro's study, the group that consumed Carioblis for 90 days showed inhibited caries progress because of the bacteriocin produced by S. salivarius M18, which can kill S. mutans. This study also showed that clinical S. salivarius (isolated from saliva and tongue) had bacteriocins that inhibited the growth of $S$. mutans.

Bacteriocins produced by clinical $S$. salivarius can be assumed to be lantibiotics (bacteriocin Class I) [18]. Bactericidal characteristics of lantibiotics are by pore formation in the bacterial membrane and inhibition of enzyme functions through interactions with their respective substrates [19]. The process of pore formation that began with nisin and other type A lantibiotics showed an efflux of ions, cytoplasmic solutes, and metabolites from susceptible cells [19]. Pores formed on cytoplasm membrane were a place where lantibiotics removed proton motive forces (PMF), and hence removing the cells that acted as an essential energy source. PMF comprises of chemical and electrical components promoting ATP formation and ions and other metabolites that accumulate through PMF-driven transport in the membrane. With PMF removed, induced bybacteriocins activity causing

Table 1: Analysis results of inhibition zone diameter of $S$. mutans XC by whole-cell protein of $S$. salivarius ATCC 13419 and clinical S. salivarius isolated from saliva and dorsum of tongue using the well-diffusion test method

\begin{tabular}{|c|c|c|c|c|}
\hline \multirow[b]{2}{*}{ Protein concentration of $S$. salivarius ATCC $13419(\mu \mathrm{g} / \mathrm{ml})$} & \multicolumn{4}{|c|}{ Culture results based on protein concentration } \\
\hline & 14.6 & 1.46 & $1.46 \times 10^{-1}$ & $1.46 \times 10^{-2}$ \\
\hline Means of inhibition zone diameter & $0 \mathrm{~mm}$ & $0 \mathrm{~mm}$ & $0 \mathrm{~mm}$ & $0 \mathrm{~mm}$ \\
\hline Concentration of $S$. salivarius clinical strain (saliva) $(\mu \mathrm{g} / \mathrm{ml})$ & 20.6 & 2.06 & $2.06 \times 10^{-1}$ & $2.06 \times 10^{-2}$ \\
\hline Means of inhibition zone diameter & $8.25 \mathrm{~mm}$ & $0 \mathrm{~mm}$ & $0 \mathrm{~mm}$ & $0 \mathrm{~mm}$ \\
\hline Concentration of $S$. salivarius clinical strain (dorsum lidah) $((\mu \mathrm{g} / \mathrm{ml})$ & 20.3 & 2.03 & $2.03 \times 10^{-1}$ & $2.03 \times 10^{-2}$ \\
\hline Means of inhibition zone diameter & $5.75 \mathrm{~mm}$ & $0 \mathrm{~mm}$ & $0 \mathrm{~mm}$ & $0 \mathrm{~mm}$ \\
\hline
\end{tabular}


Table 2: Analysis results of inhibition zone diameter of $S$. mutans XC by spent-medium protein of $S$. salivarius ATCC 13419 and clinical S. salivarius isolated from saliva and dorsum of tongue with well-diffusion test method

\begin{tabular}{|c|c|c|c|c|}
\hline \multirow[b]{2}{*}{ Protein concentration of $S$. salivarius ATCC $13419(\mu \mathrm{g} / \mathrm{ml})$} & \multicolumn{4}{|c|}{ Culture results based on protein concentration } \\
\hline & 18.1 & 1.81 & $1.81 \times 10^{-1}$ & $1.81 \times 10^{-2}$ \\
\hline Means of inhibition zone diameter & $0 \mathrm{~mm}$ & $0 \mathrm{~mm}$ & $0 \mathrm{~mm}$ & $\mathrm{~mm}$ \\
\hline Means of inhibition zone diameter & $0 \mathrm{~mm}$ & $0 \mathrm{~mm}$ & $0 \mathrm{~mm}$ & $0 \mathrm{~mm}$ \\
\hline Concentration of $S$. salivarius clinical strain (dorsum lidah) $((\mu \mathrm{g} / \mathrm{ml})$ & 27.9 & 2.79 & $2.79 \times 10^{-1}$ & $2.79 \times 10^{-2}$ \\
\hline Means of inhibition zone diameter & $0 \mathrm{~mm}$ & $0 \mathrm{~mm}$ & $0 \mathrm{~mm}$ & $0 \mathrm{~mm}$ \\
\hline
\end{tabular}

S. salivarius: Streptococcus salivarius

cell death through stopping energy consuming reactions. This process happened in Type A lantibiotics, such as nisin and salivaricin A. Unlike Type A lantibiotics, Type B lantibiotics do not form membrane pores, but these peptides work by disrupting essential enzyme activity [20].

Lantibiotic type (A or B) could not be determined in this study. However, it can be assumed that the inhibition zone formed from clinical S. salivarius on $S$. mutans because $S$. mutans are Gram-positive bacteria that only have one lipid membrane surrounded by a cell wall, and hence enabling lantibiotics to bind directly with the lipid membrane and form pores, leading to bacterial death without having to go through outer membranes, which is the case for Gram-negative bacteria. The data also showed a better inhibitory potential of clinical S. salivarius compared to S. salivarius ATCC 13419 because the inhibition zone was not formed in any of the S. salivarius ATCC concentrations. Based on one-way ANOVA and independent samples t-test results, the inhibition zones of S. salivarius ATCC 13419 were significantly different from the inhibition zones of clinical S. salivarius. This result is in line with a study conducted by Handley that showed S. salivarius HB-V5 (laboratorium strain) weakly coaggregates with Veillonella strain V1 compared to other S. salivarius strains (fibrillary and fimbriate strain) because S. salivarius HB-V5 has a lost protein or surface receptor of $S$. salivarius HB, which mediates coaggregation with Veillonella [21].

The protein inhibitory potential produced by $S$. salivarius on the growth of $S$. mutans XC was also analyzed in this study using a well- diffusion test. The proteins tested were those on whole-cell and spent mediums. The results showed formation of an inhibition zone from whole-cell proteins of clinical $S$. salivarius isolated from saliva and the tongue's dorsum. The inhibition zone's diameter was formed only on one concentration in each isolation, which was the highest one $(20.6 \mu \mathrm{g} / \mathrm{ml}$ on saliva isolation and $20.3 \mu \mathrm{g} / \mathrm{ml}$ on the dorsum of the tongue). Meanwhile, no inhibition zone was formed when there were lower concentrations. It can be assumed that the highest concentration used in this study was the minimum requirement to form inhibition zone diameters for clinical $S$. salivarius whole-cell protein. These results also showed that the inhibition zone's diameter depended on clinical $S$. salivarius whole-cell protein concentration. An inhibition zone was not formed on spent-medium protein of clinical $S$. salivarius on $S$. mutans XC (quantitative), but it was seen qualitatively. This was shown in Fig. 6 , which showed that on a spent-medium protein of $S$. salivarius isolated from saliva, formation of colonies around the well in the highest concentration $(28.2 \mu \mathrm{g} / \mathrm{ml})$ was very thin compared to the control group. The third and fourth concentration group also showed thinning compared to the control group but less than the highest concentration. Colonies grown around the protein well were thinner compared to the control group on spent-medium protein of $S$ salivarius isolated from the tongue's dorsum. This was commonly found in every concentration, but the most prominent was when the highest concentration was used $(27.9 \mu \mathrm{g} / \mathrm{ml})$. From these results, it can be assumed that inhibition zones forming depended on the spent-medium protein concentration of clinical S. salivarius.

The data showed that no whole-cell and spent-medium protein inhibition zone diameter formed with S. salivarius ATCC 13419 in all concentrations. This method was assumed to be similar to the previous methods that stated that the wild-type S. salivarius (clinical S. salivarius) was better compared to the laboratorium strain S. salivarius (S. salivarius ATCC). Data also showed that whole-cell proteins were better at inhibiting the growth of $S$. mutans XC (measurable diameter formed) compared to the spent-medium protein, which has a qualitative value of inhibition. This was in agreement with Barbour and Philip, who stated that $60-70 \%$ of bacteriocins were peptides that bound with the cell wall of their bacteria (whole-cell) while the other $30-40 \%$ were inhibitory peptides that secreted extracellularly on a liquid medium (spent medium) [22]. Therefore, this study assumed that the results from the whole-cell protein were better than the spent-medium protein because there would be more bacteriocins contained in whole-cell proteins. Inhibition zones that formed quantitative and qualitatively from the clinical $S$. salivarius protein in this study were in agreement with the study conducted by Barbour and Philip; it was also in line with a previous theory about lantibiotics being a Class I bacteriocin produced by Gram-positive bacteria. It has a bactericidal characteristic on Gram-positive bacteria by a mechanism mode of making pores on cytoplasm membrane [19]. Lantibiotics can bond directly with lipid membranes of Gram-positive bacteria because these bacteria do not have outer membranes, which Gram-negative bacteria have. Therefore, it was assumed that in this study, lantibiotics could directly form pores, leading to target bacteria cell death. However, as with the previous methods, this method could not determine the lantibiotic type produced from clinical S. salivarius. This could be because of limitations of time and materials; therefore, purification could not be carried out to obtain specific lantibiotic proteins. Further studies about the specific lantibiotic proteins produced by clinical S. salivarius isolated from healthy saliva and the tongue's dorsum are needed.

\section{CONCLUSION}

S. salivarius isolated from saliva and the tongue's dorsum can inhibit the growth of $S$. mutans, but there was no difference in the inhibitory potential between $S$. salivarius isolated from saliva and the tongue's dorsum. Proteins produced by $S$. salivarius isolated from saliva and the tongue's dorsum can inhibit the growth of $S$. mutans, but there was no significant difference in the inhibitory potential of proteins produced by $S$. salivarius isolated from saliva and the tongue's dorsum. Further studies are needed to investigate protein purification from lantibiotics produced by clinical $S$. salivarius to develop specific lantibiotics; the inhibitory potential analysis of $S$. salivarius isolated from saliva and the tongue's dorsum swabs using a positive control of S. salivarius K12 and M18; and inhibitory potential analysis of clinical S. salivarius on other pathogenic bacteria inside the oral cavity.

\section{REFERENCES}

1. Levy SB. The 2000 Garrod lecture. Factors impacting on the problem of antibiotic resistance. J Antimicrob Chemother 2002;49(1):25-30.

2. Anurogo D. Probiotik: Problematika dan progresivitasnya. Medicinus 2014;27(3):46-57

3. Aas JA, Paster BJ, Stokes LN, Olsen I, Dewhirst FE. Defining the normal bacterial flora of the oral cavity. J Clin Microbiol 2005;43(11):5721-32.

4. Haukioja A. Probiotics and oral health. Eur J Dent 2010;4(3):348-55.

5. Goldin BR, Gorbach SL. Clinical indications for probiotics: An overview. Clin Infect Dis 2008;46 Suppl 2:S96-100.

6. Fauzi AA, Shafiei Z, Baharin B, Mohd N. Isolation of lactobacillus from periodontally healthy subjects and its antimicrobial activity 
against periodontal pathogens. Sains Malays 2013;42(1):19-24.

7. Burton JP, Wescombe PA, Moore CJ, Chilcott CN, Tagg JR. Safety assessment of the oral cavity probiotic Streptococcus salivarius K12. Appl Environ Microbiol 2006;72(4):3050-3.

8. Horz HP, Meinelt A, Houben B, Conrads G. Distribution and persistence of probiotic Streptococcus salivarius K12 in the human oral cavity as determined by real-time quantitative polymerase chain reaction. Oral Microbiol Immunol 2007;22(2):126-30.

9. Kaci G, Goudercourt D, Dennin V, Pot B, Doré J, Ehrlich SD, et al. Anti-inflammatory properties of Streptococcus salivarius, a commensal bacterium of the oral cavity and digestive tract. Appl Environ Microbiol 2014;80(3):928-34.

10. Di Pierro F, Zanvit A, Nobili P, Risso P, Fornaini C. Cariogram outcome after 90 days of oral treatment with Streptococcus salivarius M18 in children at high risk for dental caries: results of a randomized, controlled study. Clin Cosmet Investig Dent 2015;7:107-13.

11. Indonesian Ministry of Health. InfoDATIN Data and Information Center of Indonesian Ministry of Health: Situasi Kesehatan Gigi dan Mulut; 2014. Available from: http://www.depkes.go.id/download. php?file $=$ download/pusdatin/infodatin/infodatin-gilut.pdf

12. Bowe WP, Filip JC, DiRienzo JM, Volgina A, Margolis DJ. Inhibition of propionibacterium acnes by bacteriocin-like inhibitory substances (BLIS) produced by Streptococcus salivarius. J Drugs Dermatol 2006;5(9):868-70.

13. Wescombe PA, Upton M, Dierksen KP, Ragland NL, Sivabalan S, Wirawan RE, et al. Production of the lantibiotic salivaricin A and its variants by oral streptococci and use of a specific induction assay to detect their presence in human saliva. Appl Environ Microbiol 2006;72(2):1459-66.

14. Prasad S, Morris PC, Hansen R, Meaden PG, Austin B. A novel bacteriocin-like substance (BLIS) from a pathogenic strain of Vibrio harveyi. Microbiology 2005;151:3051-8.

15. Samaranayake L. Essential Microbiology for Dentistry. $3^{\text {rd }}$ ed. Edinburgh: Churchill Livingstone Elsevier; 2006.

16. Wescombe PA, Hale JD, Heng NC, Tagg JR. Developing oral probiotics from Streptococcus salivarius. Future Microbiol 2012;7(12):1355-71.

17. Roger P, Delettre J, Bouix M, Béal C. Characterization of Streptococcus salivarius growth and maintenance in artificial saliva. J Appl Microbiol 2011;111(3):631-41.

18. Wescombe PA, Heng NC, Burton JP, Chilcott CN, Tagg JR. Streptococcal bacteriocins and the case for Streptococcus salivarius as model oral probiotics. Future Microbiol 2009;4(7):819-35.

19. Pag U, Sahl HG. Multiple activities in lantibiotics-models for the design of novel antibiotics? Curr Pharm Des 2002;8:815-33.

20. Chatterjee C, Paul M, Xie L, van der Donk WA. Biosynthesis and mode of action of lantibiotics. Chem Rev 2005;105(2):633-83.

21. Handley PS, Harty DW, Wyatt JE, Brown CR, Doran JP, Gibbs AC. A comparison of the adhesion, coaggregation and cellsurface hydrophobicity properties of fibrillar and fimbriate strains of Streptococcus salivarius. J Gen Microbiol 1987;133(11):3207-17.

22. Barbour A, Philip K. Variable characteristics of bacteriocin-producing Streptococcus salivarius strains isolated from Malaysian subjects. PLoS One 2014;9(6):e100541. 